\section{How to EB-ind microtubule ends}

Microtubules are polar cylindrical structures of linear protofilaments consisting of tubulin heterodimers. Microtubule ends undergo continuous assembly and disassembly powered by guanosine triphosphate (GTP) hydrolysis. The rapidly growing microtubule plus ends interact with many proteins, collectively known as plus-end tracking proteins (+TIPs). Among +TIPs, the end-binding (EB) proteins contact the growing microtubule end directly, to mediate further +TIP-microtubule interactions. Despite having been extensively studied, how EBs associate with microtubule ends has remained a long-standing question.

Maurer et al. elucidate the structural basis of the EB-microtubule interaction by employing cryo-electron microscopy, subnanometre single particle reconstruction and fluorescence microscopy (Cell 13, 371-382; 2012). They show that Mal3, the fission yeast EB, binds microtubule protofilaments by contacting four tubulin dimers. Their data indicate that the proximity of EB to the GTP-binding site of tubulin allows it to interact with the growing microtubule end by sensing conformational changes resulting from the microtubule nucleotide state. The authors further demonstrate that EB binding to the microtubule end forms a stabilizing zone that protects microtubules from depolymerization. These findings link EB function to microtubule-end stability and underscore the importance of EBs in forming a structural and functional hub at microtubule ends that supports recruitment of further factors to the microtubule plus end.

AIZ

\section{Visualizing clathrin- mediated endocytosis}

Clathrin-mediated endocytosis (CME) is a major mechanism for internalizing plasmamembrane receptors. Clathrin-coated structures (CCSs) associate with cargo and adaptor proteins, and begin to invaginate the membrane, eventually giving rise to clathrin-coated vesicles (CCVs). Merrifield and colleagues use total internal reflection fluorescence microscopy, coupled to a pulsed $\mathrm{pH}$ assay, to study the dynamics of CME machinery recruitment and generation of CCVs in mammalian cells (PLoS Biol. 9, e1000604; 2012).

Through these elegant analyses, the authors found that CCSs could persist at the plasma membrane through multiple scission events, indicating that CCS disappearance might not be a reliable marker for CCV generation. Moreover, the duration and size of CCSs at the membrane was heterogeneous. The authors also generated 'recruitment signatures' of 34 proteins involved in CME. The signatures revealed that a core collection of coat, adaptor, actinbinding and dynamin proteins are involved in most CCV scission events. Furthermore,

\title{
Pushing a way to axis determination
}

In Drosophila melanogaster oocytes, the nucleus moves from a posterior localization towards an anterior corner, which then becomes the dorsal axis. This movement was thought to be powered by the dynein motor and microtubules that are simultaneously nucleated along the anterior lateral cortex, setting up the antero-posterior axis. But Daniel St. Johnston and colleagues have now found that the nucleus is pushed away by the force provided by microtubules polymerizing from organizing centres localized behind the nucleus at the posterior, thus dissociating migration and dorso-ventral determination from antero-posterior axis set-up (Science http://doi.org/hwj; 2012).

Using live imaging, they noticed a deformation in the nucleus in the direction of movement, indicating the existence of a pushing force. They observed an enrichment of the fluorescently tagged plus-end-associated protein EB1-GFP near the indentation, and that depolymerization of microtubules decreased both the deformation and this enrichment. Further, centrosomal proteins were localized behind the nucleus, indicating that EB1-GFP-labelled microtubules grew from this location. Laser-mediated ablation of the centrosomes prevented the deformation, and ectopic anterior deformations were seen in mutants with mislocalized centrosomes. The authors calculated that the force exerted by only a few microtubules would be sufficient to drag the nucleus away from the posterior, consistent with their estimation of the number of microtubules touching the nucleus. As indentations were seen on nuclei still anchored at the posterior, the adjacent follicular cell may maintain the nuclei in place until a release signal is sent. Discovering the nature of this signal is the next challenge.

similarly to CME in yeast, CCS maturation and CCV generation proceeded in a stepwise manner, with distinct protein modules being recruited to the CCS at different times.

Thus, this high-resolution approach has yielded a temporal map of CME in mammalian cells, revealing fresh insights into this process and opening new avenues for investigation.

EJC

\section{Trim39 ligase keeps apoptosis going}

The intrinsic apoptosis pathway is mediated by Bcl-2 family proteins, such as Bax. Kornbluth and colleagues now identify a link between the anaphase-promoting complex/cyclosome (APC/C) and apoptosis by demonstrating that the modulator of apoptosis protein (MOAP-1), an enhancer of Bax function, is stabilized through Trim39-mediated inhibition of APC/C (J. Cell Biol. 197, 361-367; 2012).

The authors identify Trim39 as a human homologue of Xnf7, a Xenopus laevis E3 ligase that they previously found to inhibit the APC/C. They also demonstrate that Trim39 inhibits ubiquitylation of the APC/C substrate cyclin $\mathrm{B} 1$, indicating that this role may be conserved. MOAP-1 was previously shown to be stabilized by Trim39; this is confirmed by the current study, which shows that MOAP-1 is degraded in the G1 phase of the cell cycle. Further, the authors find that MOAP-1 is a target of the APC/C cofactor Cdh1, as mutation of D-box sequences stabilizes the protein, whereas Cdh1 overexpression promotes its ubiquitylation. In accordance with MOAP-1 being a target of APC/C-Cdh1, chemotherapy-induced activation of Bax and apoptosis is enhanced by Cdh1 depletion, in a manner at least partly dependent on MOAP-1. How the $\mathrm{APC} / \mathrm{C}$ is inhibited by Trim 39 remains unclear, but the mechanism may involve ubiquitylation of an APC/C regulator, as the catalytic activity of Trim39 is required. More work will also be needed to elucidate how MOAP-1 degradation could help coordinate apoptosis with the cell cycle.

CKR 